\title{
Orthographic vs. phonological irregularity in lexical decision
}

\author{
ALAN J. PARKIN \\ Laboratory of Experimental Psychology, University of Sussex \\ Brighton, East Sussex BNI 9QG, England \\ and \\ GEOFFREY UNDERWOOD \\ Department of Psychology, University of Nottingham, Nottingham NG7 2RD, England
}

\begin{abstract}
This experiment investigates whether the influence of spelling-to-sound correspondence on lexical decision may be due to the visual characteristics of irregular words rather than to irregularities in their phonological correspondence. Lexical decision times to three types of word were measured: words with both irregular orthography and spelling-to-sound correspondence (e.g., GHOUL, CHAOS), words with regular orthography but irregular spelling-to-sound correspondence (e.g., GROSS, LEVER), and words regular in both respects (e.g., SHACK, PLUG). Items were presented in upper- and lowercase in order to examine the influence of "word shape" on any irregularity effects obtained. The results showed that irregular words with regular orthographies were identified more slowly than regular words in both upper- and lowercase. Words that are both orthographically and phonologically irregular were identified much more slowly with lowercase presentation. However, with uppercase, the lexical decision time for these items did not differ significantly from those of regular words. These data indicate that previous demonstrations of the regularity effect in lexical decision were not due to the unusual visual characteristics of some of the words sampled. In addition, the data emphasize the role of word shape in word recognition and also suggest that words with unique orthographies may be processed differently from those whose orthography is similar to other words.
\end{abstract}

In a recent series of experiments, Parkin (1982b) has demonstrated that irregularities in spelling-to-sound correspondence have a detrimental influence on the speed at which isolated words can be identified and pronounced. More recently, it has been shown that such effects also occur when identification is facilitated by semantic context (Parkin, 1982a) and when pseudohomophones are used as distractors in the lexical decision task (Parkin \& Ellingham, in press).

Interpreting these effects, Parkin (1982b) has suggested that they occur because lexical access from the printed word involves a phonological recoding stage in which a phonemic representation is derived from its visual representation and then utilized in the access procedure. ${ }^{1} \mathrm{He}$ suggests that this recoding system is acquired through the individual's general linguistic experience and appreciation of regularities in English spelling-to-sound correspondence. Furthermore, on the basis of experimental evidence, it was argued that these rules extend beyond the level of grapheme-to-phoneme correspondence (cf. Venezky, 1970) and incorporate information about correspondences to larger ortho-

This research was partially supported by Medical Research Council Grant G/978/1173/N awarded to G. Underwood. Reprints can be obtained from both authors. graphic units. Thus the word HEAD is irregular at the grapheme-to-phoneme level (EA being most commonly pronounced [i]) but can be considered regular if the larger unit EAD serves as the basis of analysis. For the purposes of this paper, therefore, the term "irregular" will refer to words whose spelling-to-sound correspondence is exceptional when all reasonable units of analysis have been taken into account.

The present study explores another possible factor that may have contributed to the effects of spelling-tosound correspondence on lexical access. In all the studies concerned with the regularity effect (e.g., Bauer \& Stanovich, 1980; Parkin, 1982a, 1982b), irregular words were selected only in terms of their spelling-to-sound correspondence. No account was taken of the fact that many of these words were also visually unusual in that they were composed of unusual sequences of graphemes (e.g., LIQUOR, TSAR, YACHT, MAUVE, SUAVE, BERET, CHAOS, CELLO). Conversely, the regular words used in these studies tended to be composed of more familiar sequences of letters (e.g., QUICK, SLOT, SPADE, SHIN). It is therefore possible that the effects of regularity obtained in earlier experiments may have been partly or even wholly attributable to the unusual visual characteristics of the irregular words as opposed to irregularities in their spelling-to-sound correspondence. 
It therefore seemed necessary to devise an experiment in which the effects of spelling-to-sound irregularity on lexical access could be observed in the absence of any confounding influence of orthographic irregularity. In the following experiment, the effects of two types of irregularity on lexical decision time are examined. The first type of irregularity will be defined as "orthographically irregular/phonologically irregular" (OIPI) and includes words composed of unusual letter sequences and possessing an irregular spelling-to-sound correspondence (e.g., CHAOS, COLONEL). In this case, an "unusual" letter sequence is defined as one whose mispronounced letter sequence is not repeated in any other word. The second type of word is that defined as "orthographically regular/phonologically irregular" (ORPI) and includes words with common letter patterns but irregular spelling-to-sound correspondence. Thus LEVER (pronounced LEE-ver) is defined as an ORPI word because its mispronounced letter sequence occurs regularly pronounced in other words (e.g., EVER, NEVER, SEVER). Other examples of ORPI items are BALLET, BOWL, STEAK, VASE.

Underwood and Bargh (1982) have further questioned the generality of the regularity effect by suggesting that phonological mediation, upon which regularity effects are dependent, may be attenuated when fuller visual information about the word is made available. They required subjects to pronounce regular and irregular words following a congruous sentence, an incongruous sentence, or no sentence at all. For each condition, half the target words were presented in uppercase and half in lowercase. With lowercase presentation, pronunciation latencies for regualr words and irregular words did not differ, but with uppercase presentation, typical regularity effects emerged. Underwood and Bargh interpreted this result as showing that the additional "word-shape" information provided by the ascending and descending letters of lowercase provides additional visual information that is sufficient to allow lexical access on the basis of purely visual analysis. With uppercase, visual information is relatively less informative and phonological mediation occurs, thus providing the basis for the regularity effect. Since previous demonstrations of the regularity effect in lexical decision had exclusively used uppercase presentation, it seemed important to explore the influence of case on the regularity effect. Accordingly, half the subjects in the following experiment experienced uppercase presentation and the other half, lowercase presentation.

\section{METHOD}

\section{Subjects}

Thirty-two students and staff from the University of Nottingham participated as volunteers in this experiment. All had normal or corrected-to-normal vision.

\section{Materials}

Twenty-four word triplets were selected, with one OIPI item, one ORPI item, and one orthographically and phonologi- cally regular word $(R)$ in each triplet. Items within each triplet were matched for letter length, frequency, and, in most cases, syllable length and part of speech. Word frequency was examined in relation to two word counts because of possible inaccuracies that might occur when relatively low-frequency words constitute a considerable proportion of the sample (cf. Taft, 1981). Using Kucera and Francis (1967), the average frequency per million was OIPI 16.6 (SE = 6.6), ORPI 20.8 (6.9), and $\mathrm{R} 18.0$ (6.6). The second count used was that of Carroll, Davies, and Richman (1971). This more extensive work allows the calculation of a "standard word-frequency index," a measure of word frequency that takes into account the log-normal distribution of word frequency and the range of literature samples within which a given word occurs. For the present materials, the following values were obtained: OIPI $41.8(1.7)$, ORPI $44.6(1.6)$, and $R \quad 44.3$ (1.7). Seventy-two nonwords were constructed by changing one letter of a real word, with the constraint that the resulting nonword obey the rules of English orthography. A further eight words and eight nonwords were used for practice. An uppercase and a lowercase version of each item was prepared with a Kroy lettering device using 8-point Century Schoolbook type. The black-on-white word tapes were mounted in glass $24 \times 36 \mathrm{~mm}$ slides.

\section{Procedure and Apparatus}

Subjects were randomly allocated to one of two equally sized groups, with any one subject shown only uppercase or only lowercase words. The 144 items were shown in a different pseudorandom order to each subject, with the constraint that no more than four items in sequence require the same response. The slides were back-projected onto a white screen using a Carousel projector modified with a tachistoscopic shutter. Each slide was exposed for $1 \mathrm{sec}$ and preceded by a warning tone. The intertrial interval was $1.5 \mathrm{msec}$ and was response contingent. Stimulus sequencing and data logging were controlled by a Rockwell AIM microcomputer. Subjects were seated $115 \mathrm{~cm}$ from the screen, and the visual angle of the stimuli ranged between 2.5 and $3 \mathrm{deg}$.

Instructions to subjects emphasized both speed and accuracy and required subjects to decide whether each letter string was or was not a word in the English language. Subjects made their responses by pressing one of two appropriately labeled response keys. Stimuli were presented in two blocks, with a short break between blocks while the slide trays were changed. Each block of trials was preceded by eight practice trials.

\section{RESULTS}

The mean correct response latencies for each condition were calculated for each subject, and the overall means are shown in Table 1 . The mean lexical decision times for each word in the experiment can be found in the appendix.

A two-way analysis of variance was performed on these data with word type (OIPI, ORPI, R) and case of presentation (uppercase, lowercase) as fixed factors. A materials-based analysis was also undertaken to allow the calculation of min $F^{\prime}$ (Clark, 1973) for each of the effects. The analysis revealed a main effect of word type $\left[\min F^{\prime}(2,67)=3.4, p<.05\right]$. The interaction term did not reach significance with $\min F^{\prime}$ analysis, but it was significant in both subjects- and materials-based analyses [by subjects, $F(2,60)=6.45, p<.01$; by materials, $\mathrm{F}(2,46)=4.05, \mathrm{p}<.025]$.

Examination of Table 1 suggests that the interaction can be attributed to the fact that the ORPI/R difference 
Table 1

Mean Response Latencies (in Milliseconds) and Mean Error Percentages for the Three Word Types as a Function of Case of Presentation

\begin{tabular}{lrrrrrrr}
\hline & \multicolumn{3}{c}{ Lowercase Words } & & \multicolumn{3}{c}{ Uppercase Words } \\
\cline { 2 - 3 } \cline { 7 - 8 } & OIPI & ORPI & R & OIPI & ORPI & R \\
\hline Mean RT & 592 & 568 & 548 & 605 & 618 & 586 \\
SD & 66 & 65 & 65 & 67 & 83 & 77 \\
Mean Errors & 11.2 & 8.9 & 7.3 & 12.8 & 12.2 & 6.8 \\
\hline
\end{tabular}

Note-OIPI words are orthographically and phonologically irregular (e.g., BOUQUET). ORPI words are orthographically regular and phonologically irregular (e.g., MASSAGE). $R$ words are both orthographically and phonologically regular (e.g., SLIPPER).

is relatively constant as a function of case but the $\mathrm{OIPI} / \mathrm{R}$ difference is not. To examine this possibility, two further analyses of variance were carried out. The first examined the ORPI/R difference as a function of case. This revealed a main effect of word type $\left[\min F^{\prime}(1,49)=6.9\right]$ but no interaction between this variable and case of presentation. The apparent advantage of lowercase presentation was reliable by the materials analysis $[\mathrm{F}(1,23)=54.4, \mathrm{p}<.001]$ but unreliable in the by-subjects analysis $[F(1,30)=2.9$, $p>.05]$. The second analysis examined the OIPI/R difference as a function of case. The main effect of word type was significant $\left[\min \mathrm{F}^{\prime}(1,34)=6.54, \mathrm{p}<.01\right]$, and the interaction between word type and case was also significant $\left[\min \mathrm{F}^{\prime}(1,53)=3.35, .05<\mathrm{p}<.1\right.$; by subjects, $F(1,30)=5.44, p<.05$; by materials, $F(1,23)$ $=8.7, \mathrm{p}<.01]$. The main effect of case was again significant by materials $\left[\min \mathrm{F}^{\prime}(1,23)=9.38, \mathrm{p}<.01\right]$ but not significant in the by-subjects analysis $(F<1)$. To evaluate this interaction further, independent analyses of the OIPI/R difference within each case condition were carried out. For uppercase presentation, the OIPI/R difference did not approach significance in either by-materials or by-subjects analyses. With lowercase, the OIPI/R difference was extremely reliable $\left[\min F^{\prime}(1,37)=11.06, p<.01\right]$.

The error data summarized in Table 1 were also submitted to an analysis of variance, but as many words elicited no errors, the analysis was limited to that using subjects as the random factor. As would be suggested by inspection of Table 1, the effect of word type affected performance $[F(2,60)=5.43, p<.01]$, but this was in evidence more with uppercase presentations $[F(2,30)=$ $3.73, p<.05]$ than with lowercase presentation $[\mathrm{F}(2,30)=2.13, \mathrm{p}>.05]$.

\section{DISCUSSION}

This experiment produced several findings of interest. First, it is clear from these data that earlier demonstrations of the regularity effect in lexical decision (e.g., Bauer \& Stanovich, 1980; Parkin, 1982a, 1982b) did not arise because the irregular word samples were composed of a significant proportion of visually unusual words. The present results show that ORPI words took longer to identify than regular words in both upper- and lowercase presentation conditions, a finding that is consistent with the assertion that the processes underlying lexical decision involve phonological mediation.

A second important aspect of the data was the observed interaction between case of presentation and word type. This interaction can be interpreted as showing that regularity effects involving ORPI words are relatively constant as a function of case. With OIPI words, however, the regularity effect is highly significant with lowercase presentation but nonsignificant with uppercase presentation. One explanation of these data centers on the possible role that word shape could play in the process of lexical access. It can be reasonably argued that OIPI words possess unusual word shapes whose general characteristics are not shared by other words. In contrast, ORPI and R words have "familiar" shapes since, by definition, they are composed of letter sequences found in other words. For ORPI and R words, it can therefore be argued that word-shape information, because of its familiarity, serves to facilitate lexical access by providing additional confirmatory evidence of lexical status. With OIPI words, however, lowercase presentation gives rise to a word shape that is "unfamiliar" in the sense that its characteristics are not shared by other words. As a result, there is no evidence of general familiarity to facilitate recognition, and lexical access is therefore slowed down. A further implication of this argument is that one would expect ORPI words presented in lowercase to be identified faster than lowercase OIPI words since the former have the advantage of a familiar word shape. Examination of Table 1 appears to confirm this, with lowercase ORPI words, on average, being identified $34 \mathrm{msec}$ faster. A by-subjects comparison of means confirms this apparent difference $[t(15)=3.44, p<.01]$. The materials analysis, however, falls short of conventional significance $[t(15)=1.3, p>.05]$, a finding that suggests that only a particular subset of the OIPI items had word shapes that were sufficiently unfamiliar to disrupt lexical access.

A more difficult finding to account for is the absence of any difference between OIPI and $R$ words when uppercase presentation is used. One possibility is that words composed of unique letter sequences assume some form of ideographic status within the language; this might be particularly likely for words such as YACHT, in which the application of recoding rules would not easily produce a satisfactory phonemic description. With uppercase presentation, this ideographic information is not countermanded by the detrimental effects of word shape that are present with lowercase presentation. As a result, uppercase presentation allows the efficient extraction of this ideographic information, and lexical access is facilitated. 
An explanation of the present data in these terms must also be reconciled with those reported by Underwood and Bargh (1982). As noted earlier, these authors found that pronunciation latencies for irregular words were significantly longer than regular words with uppercase presentation, but no such difference was found with lowercase presentation. In terms of relative processing times, this represents a reverse of the present results. However, it might be accounted for by suggesting that factors such as word shape and orthographic uniqueness play different roles in reading tasks in which the letter string is known to be a word (e.g., pronunciation) compared with those in which lexical identity must be ascertained (e.g., lexical decision). In pronunciation, for example, unusual word shape might act as a useful cue to lexical identity only because the item is known to be a word. In this case, the visual information enables lexical access to occur without phonological mediation, and the basis for the regularity effect is removed. In this connection, it is interesting to note that $56 \%$ of the word sample used by Underwood and Bargh consists of items that can be placed in the OIPI category. With lexical decision, however, basic lexical status must be established, and it has been argued that unusual word shape inhibits this decision because the shape is perceived as one that is unfamiliar. This in turn might initiate some form of checking process before a lexical decision is made. One must also explain why uppercase presentation gives rise to a regularity effect in pronunciation but not in lexical decision. A possible answer is that pronunciation involves an interaction between two processes; a lexical "lookup" process whereby the lexical identity of the letter string is accessed and the pronunciation is derived, and a series of nonspecific pronunciation rules in which orthographic segments are assigned a phonemic code. On this assumption, OIPI words are likely to result in slower processing because their orthographic construction is unfamiliar (e.g., uncommon sequences of graphemes) and the resulting phonemic description is irregular. The net result of this would be to devalue any advantage orthographic uniqueness imparts to the process of lexical access.

The initial aim of this experiment was to establish that the regularity effect in lexical decision did not arise because previous demonstrations of this effect had used irregular word samples in which many of the items were visually unusual. This was confirmed by showing that words with irregular spelling-to-sound correspondence, but regular orthographies produced significantly longer lexical decision latencies than regular words.
Furthermore, this effect was shown to be independent of case. An unexpected aspect of the results was the interaction between OIPI words and $\mathrm{R}$ words as a function of case. The explanation we have offered above is both tentative and decidedly post hoc. Nonetheless, we feel that the possibilities raised here merit further consideration.

\section{REFERENCES}

Bauer, D. W., \& Stanovich, K. E. Lexical access and the spelling-to-sound regularity effect. Memory \& Cognition, 1980, 8, 424-432.

Carroll, J. B., Davies, P., \& Richman, B. The American heritage word frequency book. Boston: Houghton-Mifflin, 1971.

Clark, H. H. The language-as-a-fixed-effect fallacy: A critique of language statistics in psychological research. Journal of Verbal Learning and Verbal Behavior, 1973, 12, 335-359.

Glushro, R. The organization and activation of orthographic knowledge in reading aloud. Journal of Experimental Psychology: Human Perception and Performance, 1979, 5, 674-691.

KAY, J., \& MArCEL, A. One process, not two, in reading words aloud: Lexical analogies do the work of non-lexical rules. Quarterly Journal of Experimental Psychology, 1981, 33A, $397-413$.

Kučera, H., \& Francis, W. N. Computational analysis of present-day American English. Providence, R.I: Brown University Press, 1967.

Parkin, A. J. Phonological recoding and context. Current Psychological Research, 1982, 2, 187-194. (a)

Parkin, A. J. Phonological recoding in lexical decision: Effects of spelling-to-sound regularity depend on how regularity is defined. Memory \& Cognition, 1982, 10, 43-53. (b)

Parkin, A. J., \& Ellingham, R. Phonological recoding and lexical decision: The influence of pseudohomophones. Language and Speech, in press.

TAFT, M. Prefix stripping revisited. Journal of Verbal Learning and Verbal Behavior, 1981, 20, 289-297.

UNDERWOOD, G., \& BARGH, K. Word shape, orthographic regularity and contextual interactions in a reading task. Cognition, 1982, 12, 197-209.

VENEZKY, R. L. The structure of English orthography. The Hague: Mouton, 1970.

\section{NOTE}

1. There is, at present, some controversy concerning the basis by which the phonological representation of a given letter string could be derived. Some authors have suggested that phonological descriptions for both real words and nonwords are derived by lexical analogy (Glushko, 1979; Kay \& Marcel, 1981). If this is the case, effects of spelling-to-sound irregularity on lexical decision must be explained as a postlexical phenomenon. However, Parkin (1982b) has provided evidence against this view and argued instead that the regularity effect in lexical decision arises from the application of nonlexical spelling-tosound rules during the lexical access procedure.

Appendix

Materials Used in the Experiment and Mean Lexical Decision Time (in Milliseconds) for Each Item as a Function of Case

\begin{tabular}{|c|c|c|c|c|c|c|c|c|}
\hline & \multicolumn{2}{|c|}{ OIPI } & & \multicolumn{2}{|c|}{ ORPI } & & \multicolumn{2}{|c|}{$\mathbf{R}$} \\
\hline & Uppercase & Lowercase & & Uppercase & Lowercase & & Uppercase & Lowercase \\
\hline BUGLE & 655 & 653 & DEMON & 583 & 605 & MELON & 532 & 599 \\
\hline QUAY & 662 & 600 & VASE & 545 & 555 & CASK & 551 & 558 \\
\hline TSAR & 739 & 689 & COUP & 627 & 734 & THUG & 646 & 599 \\
\hline
\end{tabular}




\begin{tabular}{lllllllll} 
GHOUL & 582 & 738 & PLAIT & 681 & 657 & SHACK & 619 & 672 \\
ALLEGE & 643 & 684 & INDICT & 556 & 663 & DIVINE & 557 & 546 \\
THYME & 612 & 629 & SLOTH & 726 & 719 & LATHE & 587 & 713 \\
SCOURGE & 661 & 727 & DRAUGHT & 500 & 557 & SCREECH & 541 & 706 \\
SUEDE & 656 & 599 & CASTE & 711 & 746 & SABLE & 627 & 679 \\
BROOCH & 619 & 643 & DEARTH & 760 & 710 & THRONG & 626 & 723 \\
BOUQUET & 557 & 652 & MASSAGE & 510 & 679 & SLIPPER & 512 & 583 \\
SPINACH & 563 & 596 & PREMISE & 501 & 638 & TREASON & 594 & 552 \\
EPOCH & 666 & 686 & LOSER & 618 & 580 & CLICK & 550 & 556 \\
LIQUOR & 548 & 549 & BALLET & 546 & 566 & MUTTON & 550 & 620 \\
CHOIR & 487 & 540 & FEVER & 512 & 566 & TOKEN & 542 & 564 \\
AISLE & 626 & 575 & STEAK & 510 & 585 & SPARK & 528 & 522 \\
GAUGE & 693 & 595 & COUGH & 539 & 574 & GRILL & 529 & 565 \\
CHAOS & 575 & 599 & LEVER & 526 & 619 & VOCAL & 510 & 546 \\
REGIME & 601 & 601 & PRAYER & 516 & 597 & MOTIVE & 505 & 508 \\
ACHE & 517 & 515 & BOWL & 526 & 547 & PLUG & 499 & 512 \\
YACHT & 550 & 531 & BOUGH & 615 & 614 & FLUTE & 546 & 577 \\
SUBTLE & 616 & 597 & STEADY & 528 & 577 & INSIST & 552 & 609 \\
COLONEL & 536 & 559 & CHAMBER & 505 & 658 & FEATURE & 520 & 561 \\
GUARD & 485 & 531 & GROSS & 644 & 647 & CROSS & 504 & 622 \\
ANSWER & 542 & 493 & POLICE & 506 & 519 & MARKET & 493 \\
\hline
\end{tabular}

Note-OIPI words are orthographically and phonologically irregular (e.g., BOUQUET). ORPI words are orthographically regular and phonologically irregular (e.g., MASSAGE). $R$ words are both orthographically and phonologically regular (e.g., SLIPPER). American readers should note that in England VASE rhymes with CARS. Also, LEVER is pronounced as in "Lever Brothers."

(Manuscript received April 28, 1982;

revision accepted for publication December $15,1982$. 